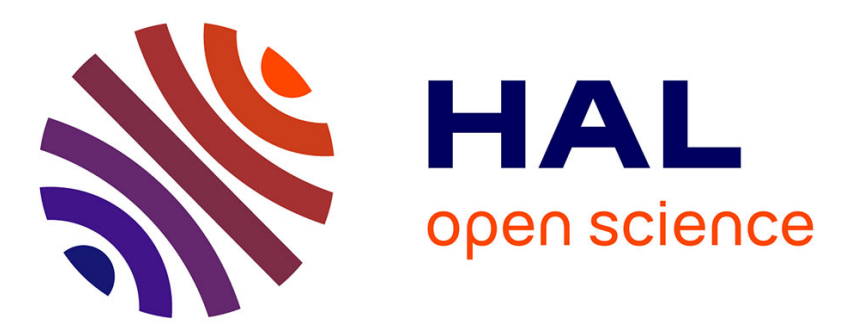

\title{
Optical Parametric Oscillators based light source for Coherent Raman Scattering microscopy: a practical overview
}

S. Brustlein, Patrick Ferrand, N. Walther, S. Brasselet, C. Billaudeau, D. Marguet, H. Rigneault

\section{To cite this version:}

S. Brustlein, Patrick Ferrand, N. Walther, S. Brasselet, C. Billaudeau, et al.. Optical Parametric Oscillators based light source for Coherent Raman Scattering microscopy: a practical overview. Journal of Biomedical Optics, 2011, 16, pp.021106. 10.1117/1.3533311 . hal-00517259

\section{HAL Id: hal-00517259 \\ https://hal.science/hal-00517259}

Submitted on 23 Apr 2015

HAL is a multi-disciplinary open access archive for the deposit and dissemination of scientific research documents, whether they are published or not. The documents may come from teaching and research institutions in France or abroad, or from public or private research centers.
L'archive ouverte pluridisciplinaire HAL, est destinée au dépôt et à la diffusion de documents scientifiques de niveau recherche, publiés ou non, émanant des établissements d'enseignement et de recherche français ou étrangers, des laboratoires publics ou privés. 


\title{
Optical parametric oscillator-based light source for coherent Raman scattering microscopy: practical overview
}

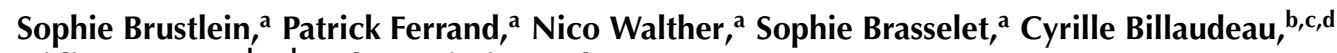 \\ Didier Marguet, ${ }^{b, c, d}$ and Hervé Rigneault ${ }^{\mathrm{a}}$ \\ ${ }^{a}$ Aix-Marseille Université, CNRS, Institut Fresnel, Ecole Centrale Marseille, Campus de Saint Jérôme, Marseille, \\ 13397 Cedex 20, France \\ ${ }^{b}$ Centre d'Immunologie de Marseille-Luminy, INSERM, UMR-S 631, Marseille, 13009 France \\ ${ }^{\mathrm{c} C N R S, ~ U M R ~ 6102, ~ M a r s e i l l e, ~} 13009$ France \\ ¿Université de la Méditerranée, UM 631, Marseille, 13009 France
}

\begin{abstract}
We present the assets and constraints of using optical parametric oscillators (OPOs) to perform point scanning nonlinear microscopy and spectroscopy with special emphasis on coherent Raman spectroscopy. The difterent possible configurations starting with one OPO and two OPOs are described in detail and with comments that are intended to be practically useful for the user. Explicit examples on test samples such as nonlinear organic crystal, polystyrene beads, and fresh mouse tissues are given. Special emphasis is given to backgroundfree coherent Raman anti-Stokes scattering (CARS) imaging, including CARS hyperspectral imaging in a fully automated mode with commercial OPOs. @ 2011 Society of Photo-Optical Instrumentation Engineers (SPIE). [DOI: 10.1117/1.3533311]
\end{abstract}

Keywords: nonlinear microscopy; coherent Raman anti-Stokes scattering; second harmonic generation; optical parametric oscillators; medical imaging; hyperspectral imaging.

Paper 10334SSPR received Jun. 16, 2010; revised manuscript received Sep. 8, 2010; accepted for publication Sep. 13, 2010; published online Feb. 25, 2011.

\section{Introduction}

It is nowadays well admitted that one of the major drawbacks of nonlinear microscopy implementation lies in the complexity of the optical source. This is of particular importance for coherent anti-Stokes Raman spectroscopy (CARS) because this four-wave mixing process requires two independent wavelengths delivered as two temporally synchronized pulse trains. Less than ten years ago, early CARS laser sources were based on two Ti : saphire lasers temporally synchronized thanks to a dedicated electronic. ${ }^{1}$ Although still commercially available, such complex systems are expensive and often subjected to a slow temporal drift that requires care to assure temporal overlapping between the two CARS beams, namely, the pump and Stokes beams. Less expensive, more reliable, and intrinsically temporally synchronized are the optical parametric oscillators (OPOs)-based CARS systems that can routinely deliver the desired pump and Stokes beams whose frequency difference can cover the full molecular vibrational spectrum. First introduced in CARS microscopy by Evans et al., ${ }^{2}$ the current most used OPO system has not significantly evolved since the seminal work by Ganikhanov et al., ${ }^{3}$ which has paved the way to turnkey systems that are now commercially available. The latter are based on a picosecond mode-locked frequencydoubled Nd:YVO-laser $(532 \mathrm{~nm})^{4}$ that synchronously pumps an OPO cavity. The current systems provide the user with up to three independent wavelengths that can be further recombined for coherent Raman spectroscopy (CRS), including CARS and

Address all correspondence to: Hervé Rigneault, Aix-Marseille Université, CNRS, Institut Fresnel, Ecole Centrale Marseille, Campus de Saint Jérôme, Marseille, 13397 Cedex 20, France. Tel: 334912880 49; E-mail: herve.rigneault@fresnel.fr. stimulated Raman spectroscopy (SRS). The OPO itself utilizes a temperature-tuned $\mathrm{LBO}\left(\mathrm{LiB}_{3} \mathrm{O}_{5}\right)$ crystal to generate two temporally synchronized picosecond pulse trains, namely, the signal and idler beams, tunable within the 690-990 nm and 1150-2450 nm ranges, respectively. ${ }^{5}$ The additional fundamental Nd:YVO picosecond pulse train at $1064 \mathrm{~nm}$ is also temporally synchronized with the OPO and can advantageously be used as a third IR beam.

OPO systems delivering picosecond pulse trains have been successfully used to perform lipid CARS imaging in skin, ${ }^{2}$ brain, ${ }^{6} \mathrm{C}$ - elegans, ${ }^{7}$ and fish gills. ${ }^{8}$ OPOs have also been used to image chemical compounds in hair, ${ }^{9}$ and myelin, ${ }^{10}$ to detect structural features of early stage atherosclerotic plaque, ${ }^{11}$ or to monitor dissolution of an oral solid dose. ${ }^{12}$ Recently picosecond OPOs have been shown to be the ideal optical source to demonstrate stimulated Raman spectroscopy (SRS). ${ }^{13}$

Femtosecond OPO systems have also been implemented in CARS with chirped pulses to improve the spectral resolution ${ }^{14}$ and because they nicely combine with Ti:saphire oscillators to perform third harmonic generation (THG) and second harmonic generation (SHG) together with two-photon excited fluorescence (TPEF). ${ }^{15}$ Nevertheless, in a simple and direct implementation, such Femtosecond systems sufter from the poor spectral resolution necessary to address sharp vibrational band as found in biological systems and exhibit poor sensitivity as compared to picosecond systems. ${ }^{16}$

Because picosecond-based OPO systems are now becoming the more versatile and reliable optical source for CRS, it is important to present to new users the assets and constraints of

1083-3668/2011/16(2)/021106/10/\$25.00 @ 2011 SPIE 
these systems in a practical overview. In this framework, this paper is organized as follows. After presenting the material and methods used throughout this work, we concentrate on using one OPO to perform nonlinear imaging, including CRS. The difterent possible configurations are described in detail and with comments that are intended to be practically useful for the user. Explicit examples on test samples, such as nonlinear organic crystal, polystyrene beads, and fresh mouse tissues, are given. We then move in a final section to a more exotic configuration using two OPOs that can generate background-free CARS images. Special emphasis is given to CARS hyperspectral imaging in a fully automated mode with commercial OPOs.

\section{Materials and Methods}

\subsection{Samples}

\subsubsection{TTB organic crystals}

Molecular crystal test samples used for SHG characterization are 1,3,5-tricyano-2,4,6-tris(p-diethylaminostyryl) benzene (TTB). These crystals emit a strong SHG radiation due to their noncentrosymmetric molecular crystalline arrangement. TTB crystals are also emitting a strong TPEF signal for any incoming wavelengths in the near-infrared (NIR). Details of TTB free-cast film synthesis and characterization can be found in Ref. 17.

\subsubsection{Polystyrene microspheres}

Polystyrene microspheres (refractive index 1.6) of diameter 20 $\mu \mathrm{m}$ were taken as purchased from Sigma-Aldrich (St Louis, Missouri), diluted in pure water and dispersed on a cleaned microscope coverslip (refractive index $n=1.51$, thickness 150 $\mu \mathrm{m})$. Slowly drying the water solvent overnight is a simple and efficient way to ensure electrostatic adhesion between the microsphere and the glass slide. The polystyrene beads are embedded in an aqueous refractive index-matching liquid (Cargille Immersion Liquid Code OHZB, $n=1.556$ at $25^{\circ} \mathrm{C}$, Cargille Labs, Cedar Grove, New Jersey) that has also the property to give a low and spectrally flat nonresonant CARS signal. This index matching-liquid has no chemical influence on the polystyrene beads.

\subsubsection{Skin mouse slide preparation}

Mice were housed under specific pathogen-free conditions. Tissue samples of mouse ear skin were directly observed by CARS microscopy without preparation. Midback mouse skin samples were immediately embedded in optimal cutting temperature medium and frozen. Then, $20-\mu \mathrm{m}$-thick transversal cryosections were placed on glass substrate and stored at $-20^{\circ} \mathrm{C}$ until acquisition. CARS imaging was performed when the samples had thawed at room temperature. All experiment procedures were approved by the Committee on the Ethics for Animal Experimentation, in accordance with French and European directives.

\subsubsection{Olive oil}

Fresh pressed olive oil was taken as purchased from a farmer producer (Mouries, France).

\subsection{Optical Parametric Oscillator System}

The master oscillator is a picosecond mode-locked frequencydoubled Nd: YVO-laser (HighQ laser ${ }^{4}$ ) operating at $532 \mathrm{~nm}$. A second amplification stage enables the laser to deliver $8 \mathrm{~W}$ of green output power that is equally divided to pump two OPOs.

Each individual OPO (Emerald, APE, Berlin, Germany ${ }^{5}$ ) uses a temperature-tuned, noncritically phase-matched LBO $\left(\mathrm{LiB}_{3} \mathrm{O}_{5}\right)$ crystal for parametric downconversion. The temperature of the crystal sets the wavelengths of the two generated NIR signal and idler. Precise wavelength tuning is accomplished through the use of a stacked Lyot filter positioned in the OPO cavity at Brewster's angle. Because the LBO crystal is noncritically phasematched, the signal and idler beams exit the laser cavity in a collinear fashion, with pulse trains that are temporally synchronized. It is nevertheless possible to spatially separate the signal and idler beams with a supplied beam splitter. In this case, the two exits of the OPO cavity are operated. The OPO can be easily and continuously tuned over a wide spectral range from 690 to $990 \mathrm{~nm}$ for the signal and between 1150 and $2450 \mathrm{~nm}$ for the idler output.

\subsection{Optical Parametric Oscillator Control Software}

The controller of the OPO gives full diagnostics of the system (power, spectrum, etc.). Moreover, a set of commands enables the control of the signal wavelength (and idler) over three parameters. The coarse and fine tunings are gained via the temperature of the crystal and the tilt of the Lyot filter, respectively. The cavity length insures the synchronous pumping of the OPO cavity. The system can be manually set to any desired signal wavelength with a commercial user-friendly interface. Automated wavelength scanning has been performed through a home-built software to facilitate acquisition of broadband CARS spectra. First, a so-called probe program iteratively investigates the working parameters for the OPO on the desired spectral range. Working parameters include the crystal temperature, the Lyot filter tilt, and the cavity length (piezostage position). They are subsequently saved into a file, which constitutes a database of accessible wavelengths and corresponding OPO settings. Then, the main CRS microscope-driving software loads this database and applies the parameters therein into the OPO. The probe program works as follows. Temperature is adjusted to reach a sufficient OPO power, and then both Lyot filter and piezostage are optimized to find the typical parameters for each signal wavelength. The whole database (corresponding to $400-3400 \mathrm{~cm}^{-1}$ ) is created in a few hours. As described in Sec. 5 , it takes typically $60 \mathrm{~min}$ to obtain a spectrum on the $900-3200 \mathrm{~cm}^{-1}$ range with $10-\mathrm{cm}^{-1}$ resolution.

\subsection{Nonlinear Scanning Microscope}

Our custom nonlinear microscope is arranged around a commercial microscope stand (Nikon Eclipse Ti-U). The pump and Stokes beams necessary for CRS microscopy are temporally synchronized, recombined, and sent onto a couple of galvanometric scanning mirrors (6200 H, Cambridge Technology). These excitation beams enter the microscope through the backward excitation port and are directed onto the filter cube. A telescope with a magnification of 3 is used to image the scanning mirrors onto the rear aperture of the infinity-corrected focusing 
microscope objective. With this arrangement, the microscope provides diffraction-limited resolution.

Forward detection is performed thanks to a dedicated mechanical unit manufactured by Tokyo Instruments (Japan) that directly bounds to the Nikon Eclipse stand. This unit insures a three-axis manual control for the forward collection. The forward emitted light is collected using a long working distance $40 \times$ air objective (LUCPLFLN, 40×, 0.6 NA, Olympus) and focused on a channel photomultiplier (CPM) tube (Perkin Elmer Optoelectronics, CPM C972). The backward emitted light (EpiCARS) is collected by the focusing objective lens and focused on a photomultiplier (Perkin Elmer Optoelectronics, CPM C972) or on a compact spectrometer (Ocean Optics, USB 4000 VIS NIR), both connected on side ports. Note that both forward and backward detection work in the so-called nondescanned geometry and therefore must have large active areas. The magnification of the imaging optics has been carefully chosen to image on the detectors a scanning area as large as $200 \times 200 \mu \mathrm{m}^{2}$. Forward and backward CPMs work here in the photon-counting regime. This is an advantage when low signals are detected but care must be taken when strong CARS signals are detected as (i) it limits the maximum count rate to the repetition rate of the lasers and (ii) photomultipliers are subjected to nonlinearities at high count rates. In the present case, we always work well below these spurious regimes by reducing the incident laser power. A set of highand low-pass wavelength filters are used that ensure that the detected signal is free of laser background and is solely associated to the desired contrast mechanism (TPEF, SHG, or CARS).

The dual-axis driver (Micromax 67320, Cambridge Technology) of the galvanometric mirrors is controlled by analog voltages generated by a high-speed analog output PCI board (NI 6731, National Instruments). The TTL pulses generated by both forward and backward CPMs are recorded by a PCI counting board (NI 6602, National Instruments). A detailed description of the synchronisation method is given in Ref. 18. The whole system is connected to a personal computer and controlled by a home-built program (LABVIEW, National Instruments).

\section{CRS with one Optical Parametric Oscillator}

Working with a single OPO is the most common and easiest way to implement nonlinear microscopy. In this "one OPO configuration", the OPO itself provides the signal and idler beams, collinear or not, while the master oscillator provides the fundamental beam at $1064 \mathrm{~nm}$ (namely "fundamental $1064 \mathrm{~nm}$ " in the following paragraphs). There are nevertheless various ways of operating such a system, with respective assets and constraints. We shall review in this section these modes of operation.

\subsection{Collinear Signal and Idler Optical Parametric Oscillator Output}

This is the easiest way to perform CARS with one OPO. The signal and idler pulse trains are collinear, overlapped, in time, and used as the pump and Stokes wavelengths, respectively, for the CARS process. Figure 1(a) gives the schematic of this CARS setup, where the signal and idler beams are directly sent on the galvanometer mirrors. Because these two beams are collinear, power adjustment is performed simustaneously on the two colors, which is far from being optimal. Neutral density filters or
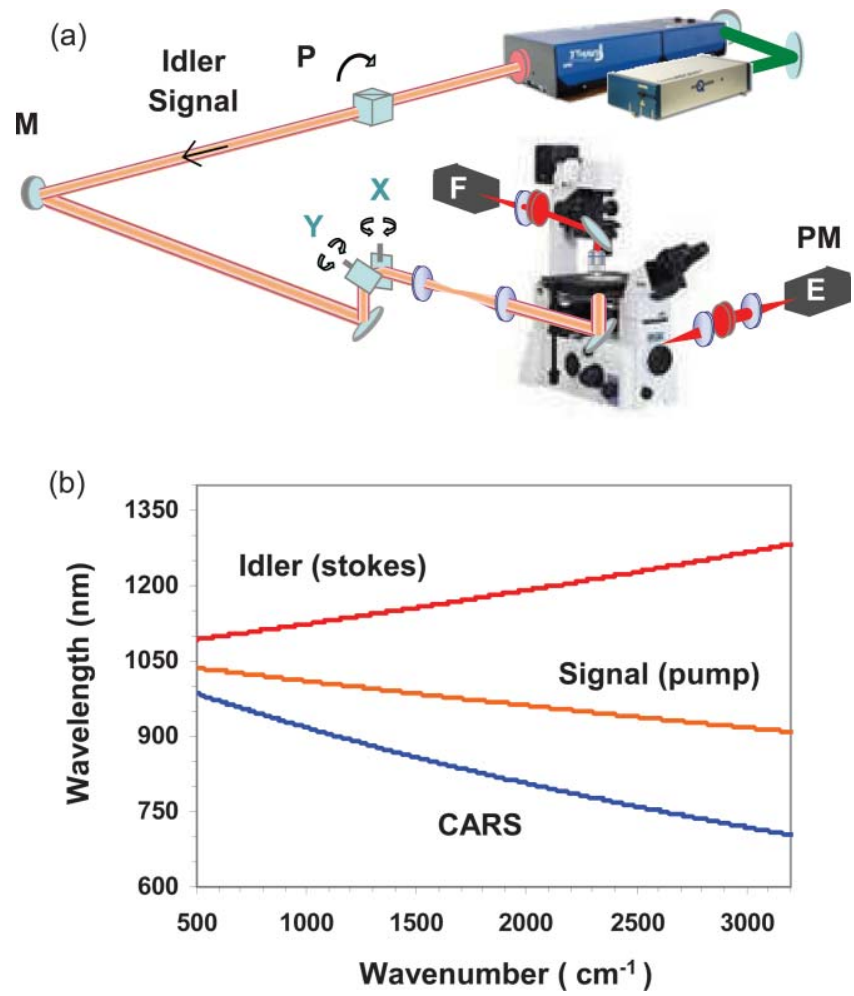

Fig. 1 (a) Simplest CARS setup using one OPO. The signal and idler trains are collinear, overlapped in time, and used as the pump and Stokes wavelengths, respectively. F and E stand for Forward and Epi (backward) detection; PM for photomultiplier tubes; $M$ for silver mirrors, and P for polarizer. (b) Signal, idler, and anti-Stokes CARS wavelengths associated with the various Raman shift.

a polarizer (if polarization at the focal point is not an issue) can be used. Because of Gaussian beam diffraction, the divergences associated with the two colors at the OPO output are not the same and impossible to tune separately. Figure 1(b) gives the signal and idler wavelength associated to each Raman shift together with the corresponding anti-Stokes CARS wavelength. The CARS wavelength is $>850 \mathrm{~nm}$ for Raman shifts of $<1500 \mathrm{~cm}^{-1}$, a spectral range where the sensitivity of standard PMT drops..$^{19}$ On the opposite, for large Raman shifts, the CARS wavelength is $<800 \mathrm{~nm}$ and can be thus easily detected. However, the associated idler beam falls in a spectral range $(>1100 \mathrm{~nm})$ where a standard microscope objective lens exhibits a poor transmission. Note that for deep-tissue penetration, IR wavelengths are less absorbed and scattered, a situation that is in favor of this collinear signal and idler configuration.

As a simple illustration of the problem that one may face using a collinear signal and idler, we focus here on longitudinal chromatic aberrations. Chromatic (and, to a lower extent, geometrical) aberrations are present in any imaging system and are difficult to control over a broad spectral range. This is an issue in CRS because the pump and Stokes beams must overlap in space, ideally for any Raman shift. It is interesting to note that the collinearity is extremely good between the signal and idler beams and, as far as we could notice, the two beams always overlap in the transverse plane $(x, y)$ at the microscope objective focus. On the contrary, this is not always the case along the optical axis ( $z$-axis). To illustrate this point, we have used the TPEF 
(a)

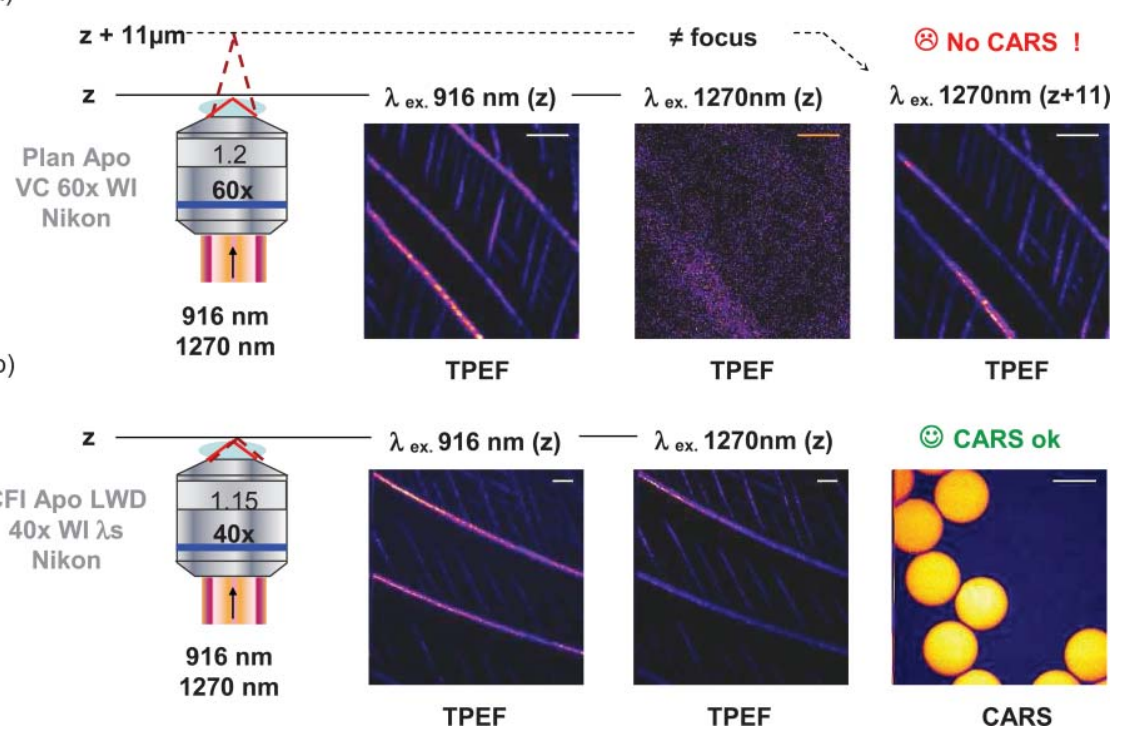

Fig. 2 Chromatic aberrations in CRS imaging using the OPO with collinear idler and signal output. (a) TPEF TTB crystal imaging at $\lambda_{\text {exc }}=916 \mathrm{~nm}$ (signal) and $\lambda_{\text {exc }}=1270 \mathrm{~nm}$ (idler) showing a difference of $11 \mu \mathrm{m}$ in the focal planes of the Nikon Plan ApoVC $60 \times$ WI objective lens. TPEF image is restored for the idler beam moving the focal plane by $11 \mu \mathrm{m}$. (b) Using the Nikon CFI Apo LWD 40× WI $\lambda_{S}$ objective lens, the two focal planes nicely overlap and allow contrasted CARS imaging of polystyrene beads at $3045 \mathrm{~cm}^{-1}$ Scale bar is $20 \mu \mathrm{m}$. Laser power was $5 \mathrm{~mW}$ for both pump and Stokes.

signal generated by TTB crystals (see Sec. 2) to check the signal and idler focal plane along the $z$-axis (alternatively, one could use fluorescent beads and two-photon absorption). TTB crystals exhibit fine spatial structures above and below the diffraction limit that nicely shows the imaging properties of a specific objective lens. Figure 2(a) shows the result using a $60 \times$ waterimmersion Plan Apo (Plan ApoVC, 60×, 1.2 NA, WI, Nikon) objective lens manufactured by Nikon. Sending the collinear idler and signal beams, we find a difference of $11 \mu \mathrm{m}$ between the focal planes of the two beams. Optimizing the TPEF image obtained with the $\lambda_{\text {exc }}=916 \mathrm{~nm}$ signal results in a poor TPEF image obtained with the $\lambda_{\mathrm{exc}}=1270 \mathrm{~nm}$ idler (corresponding to a $3045-\mathrm{cm}^{-1}$ Raman shift). By moving the focal plane $11 \mu \mathrm{m}$ farther, the idler image can be restored. With such an objective lens, it is clear that no CRS imaging is possible in this collinear configuration. Using the recently released $40 \times$ water-immersion CFI Apo (CFI Apo LWD, 40×, 1.15 NA, WI $\lambda_{\mathrm{S}}$, Nikon) objective lens manufactured by Nikon gives very different results, as can be seen in Fig. 2(b). With this well-corrected objective lens, the $\lambda_{\text {exc }}=916 \mathrm{~nm}$ signal and $\lambda_{\text {exc }}=1270 \mathrm{~nm}$ idler focal planes nicely overlap, as can be seen from the TTB crystal fine structure. Therefore, we could directly perform CARS imaging of polystyrene beads on resonance [see bottom right image in Fig. 2(b)].

When dealing with chromatic aberrations, it is possible to separate the signal and idler beams at the OPO exit to manage their divergence separately via two independent telescopes in order to match their focal planes (see Sec. 3.2). Nevertheless, this scheme can be used only for small focal-plane mismatches and rapidly proves to be very inefficient in terms of image quality. Transverse chromatic aberration may also play a role especially near the edges of the image and consequently produces intensity falloff. In our case and with the objectives used in this work, we have not noted such an effect over the $200 \times 200 \mu \mathrm{m}^{2}$ field of view. Table 1 summarizes the assets and constraints of using an OPO with a collinear signal and idler output.

We shall now move to another well-used mode of operation when only the OPO's signal and the $1064 \mathrm{~nm}$ output from the $\mathrm{Nd}: \mathrm{YVO}$ are used.

\subsection{Using the Optical Parametric Oscillator Signal and the 1064-nm Beam from the Nd:YVO Oscillator}

Another advantageous mode of operation for CRS is to use the OPO signal beam from the OPO and the fundamental 1064$\mathrm{nm}$ beam from the Nd: YVO oscillator as the pump and Stokes beams, respectively, for the CARS process. Figure 3(a) shows the schematic of the setup where only the signal from the OPO is used (the idler beam is stopped within the OPO housing with a dedicated filter) and is further recombined with the fundamental 1064-nm beam that does not enter the green module (SHG unit incorporated into the $\mathrm{Nd}$ : YVO housing).

Because the paths of the two beams are now well separated, power and divergence correction are adjusted independently with "halfwave plate/polarizer" units and telescopes, respectively. Spatial overlap between the two beams is controlled with a gimbal mirror mount while temporal overlap adjustment requires a dedicated delay line (mounted on the fundamental beam in our setup). In this configuration, it is possible to control the polarization state of the two beams independently (note that: if a retroreflector is used in the delay line, the outcome polarization state can be rotated). Figure 3(b) gives the fundamental and signal wavelength associated with the Raman shift together with the corresponding anti-Stokes CARS wavelength. The CARS wavelength falls in a range that can be detected with photomultiplier tubes if the targeted Raman shift is $>1000 \mathrm{~cm}^{-1}$. The fundamental and signal beams are now well transmitted by most of the commercial IR-corrected objective lenses. 
Brustlein et al.: Optical parametric oscillator-based light source for coherent Raman scattering microscopy: practical overview

Table 1 Pros and cons for "collinear signal and idler OPO output."

\begin{tabular}{l} 
Collinear signal and idler OPO output \\
\hline Pros: \\
Cost effective \\
Simple to implement \\
Overlap in time for the idler and signal beams \\
Transverse overlap in space $(x, y)$ for the idler and signal beams \\
NIR pump and Stokes wavelengths suitable for deep-tissue imaging \\
Cons: $\quad$ Coarse relative power adjustment for the idler and signal beams \\
Impossible relative polarization state control for the idler and signal beams \\
Possible poor idler beam transmission through the objective for Raman shifts $>1500 \mathrm{~cm}^{-1}$ \\
Fixed relative divergences for the idler and signal beams \\
Weak to zero CARS detection for Raman shifts $<1500 \mathrm{~cm}^{-1}$ (using PMTs) \\
Possible strong chromatic aberrations that prohibit CRS imaging \\
Necessity to filter out the CARS signal generated in the OPO
\end{tabular}
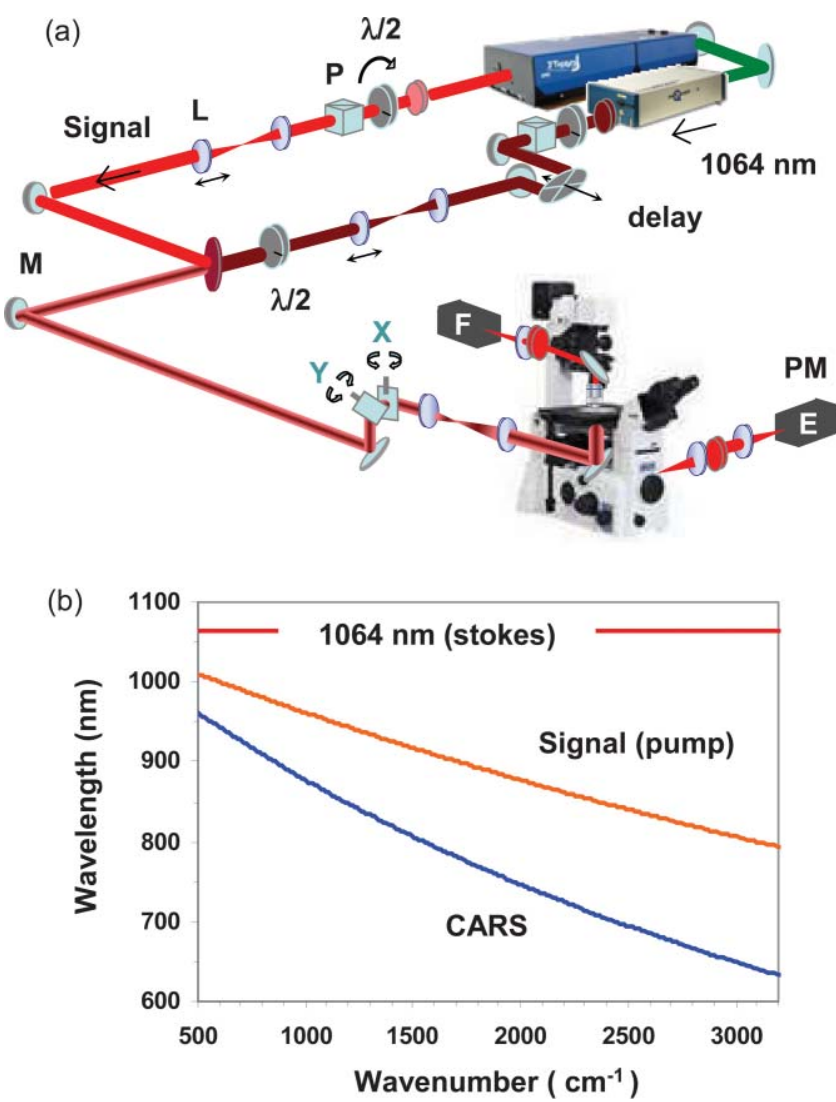

Fig. 3 (a) Efficient CARS setup using one OPO. The fundamental 1064$\mathrm{nm}$ and OPO signal pulse trains are recombined and used as the pump and Stokes beams, respectively. F and E stand for Forward and Epi detection; PM for photomultiplier tubes; $\mathrm{M}$ for silver mirrors; $\mathrm{P}$ for polarizer, and L for NIR achromatic doublets. (b) Fundamental 1064$\mathrm{nm}$, OPO signal, and anti-Stokes CARS associated with the various Raman shift.
As an example of the effectiveness of this operation mode, we report fresh mouse ear imaging on the $\mathrm{CH}_{2}$ symmetric stretching mode $\left(2845 \mathrm{~cm}^{-1}\right)$ of lipids with a $40 \times$ long working distance standard air objective lens manufactured by Olympus (LUCPL FLN, 40×, 0.6 NA, air, Olympus). Because this lens is not fully corrected in the IR, we had to slightly tune the beam divergence to match the focal planes [see Fig. 4(a)]. They were distant by $3 \mu \mathrm{m}$ when injecting parallel incoming beams. Figure 4(b) presents the forward-detected CARS signal for various penetration depths corresponding to the stratum corneum $(z=0 \mu \mathrm{m})$, the sebaceous gland $(z=30 \mu \mathrm{m})$ and the deep adipocytes of the dermis $(z=55-85 \mu \mathrm{m})$.

This "OPO signal and fundamental $1064 \mathrm{~nm}$ " configuration is certainly the best trade-off when using a single OPO (see Table 2). Such a configuration has proved to be quite versatile to perform CRS imaging in various configurations, including CARS (Ref. 6) and SRS. ${ }^{13}$ Note that hyperspectral imaging can also be done in this configuration (see Sec. 5). With the current available detectors, this is the operation mode that we recommend. We shall now move to a more exotic, nevertheless, very powerful operation mode using two OPOs.

\section{CRS with Two Optical Parametric Oscillators}

The use of three colours in CARS imaging has already been reported to perform timeresolved CARS (Ref. 20), to improve the sensitivity ${ }^{21}$ or to cover the full vibrational Raman spectrum. ${ }^{7}$

A possible setup is presented in Fig. 5 where the frequency doubled Nd:YVO generates $8 \mathrm{~W}$ of green light $(532 \mathrm{~nm})$ and pumps two OPOs with $4 \mathrm{~W}$ each. Although the full system can provide up to five wavelengths simultaneously (signal and idler for each OPO plus the fundamental $1064 \mathrm{~nm}$ ), we use three colours only in this work. The two signals coming from each $\mathrm{OPO}$ are recombined with the fundamental $1064 \mathrm{~nm}$ beam from 
(a)

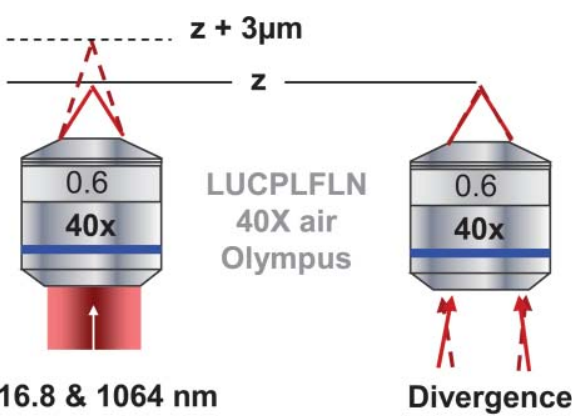

(b)
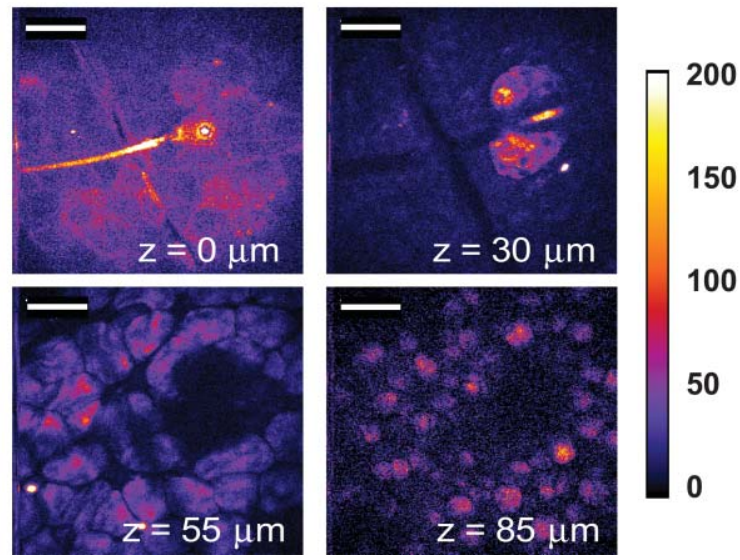

Fig. 4 (a) $z$ overlap in the focal plane using a conventional long working distance lens (LUCPL FLN $40 \times 0.6 \mathrm{NA}$ air Olympus) is possible by slightly correcting the incoming beam divergence. (b) CARS images at $2845 \mathrm{~cm}^{-1}$ of freshly removed mouse ear sample at different penetration depths: stratum corneum $(z=0 \mu \mathrm{m})$, sebaceous gland $(z=30 \mu \mathrm{m})$, and deep dermis adipocytes $(z=55-85 \mu \mathrm{m})$. Laser powers were $15 \mathrm{~mW}$ for the Stokes and $25 \mathrm{~mW}$ for the pump. Scale bar is $40 \mu \mathrm{m}$; pixel dwell time is $50 \mu \mathrm{s}$. To improve the signal-to-noise ratio, we average 10 frames of $250 \times 250$ pixel images.

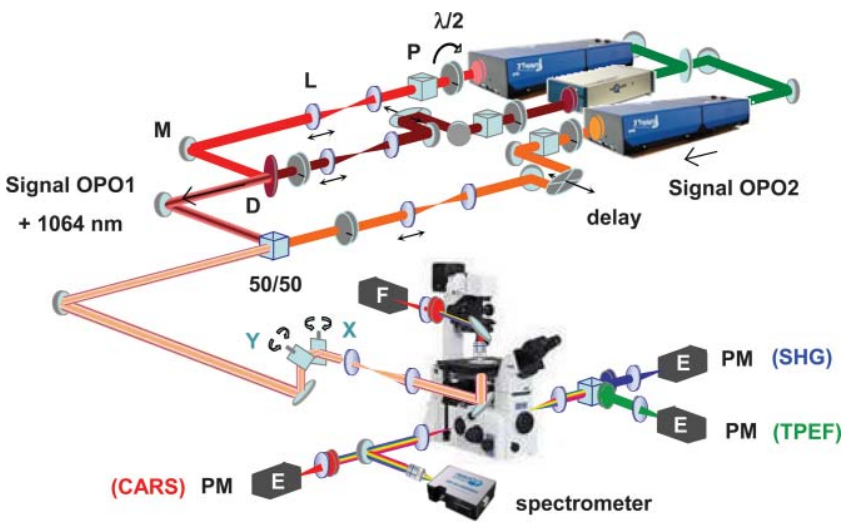

Fig. 5 CRS with two OPOs. F and E stand for Forward and Epi (backward) detection; $\mathrm{PM}$ for photomultiplier tubes; $\mathrm{M}$ for silver mirrors; $\mathrm{P}$ for polarizer, and L for NIR achromatic doublets.

the Nd: YVO oscillator. As previously, the power, polarization state and divergence of each beam are controlled independently with dedicated polarization optics and telescopes. Delay lines on two of the beam paths (signal and $1064 \mathrm{~nm}$ ) assure temporal overlap between the three beams. The backward detection is achieved (as compared to Figs. 1 and 3) with either two PMTs (that detect SHG and TPEF) or a spectrometer plus one PMT (that detects CARS). A set of dedicated filters are placed before the detectors to select the relevant spectral domains.

\subsection{Wave Mixing as Multiple Contrast Mechanisms Imaging}

When coherent Raman, sum frequency generation (SFG), SHG, and TPEF can be addressed on the same sample with three incoming colors, quite a few wave-mixing lines appear. Figure 6(a) summarizes the wave-mixing combinations that generate CARS, SFG, SHG, and TPEF. These wave-mixing combinations are readily observed on TTB crystals that provides a

Table 2 Pros and cons for "OPO signal and 1064 nm fundamental."

\begin{tabular}{ll}
\hline & OPO signal and $1064 \mathrm{~nm}$ fundamental \\
\hline Pros: & Cost effective \\
& Fine relative power adjustment for signal and fundamental beams \\
& Fine relative polarization state control for signal and fundamental beams \\
& Fine relative divergence control for signal and fundamental beams \\
& Good signal and fundamental beam transmission through objective lenses \\
& Hyperspectral imaging (see Sec. 5) \\
& Must control the temporal overlap for the signal and fundamental beams \\
& Must control the transverse overlap in space $(x, y)$ for signal and fundamental beams \\
& Weak to zero CARS detection for Raman shift $<1000 \mathrm{~cm}^{-1}$ (using PMTs) \\
& Possible remaining chromatic aberration that can be compensated, nonetheless, with the beam divergence
\end{tabular}


(a)

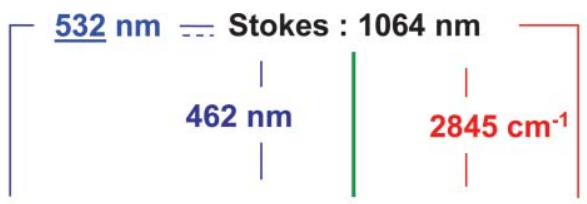

$484 \mathrm{~nm} \quad \underline{408} \mathrm{~nm}$-- OPO1:816.8 nm $1855 \mathrm{~cm}^{-1}$

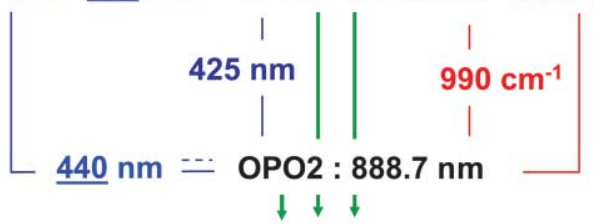

SFG SHG TPEF

\section{CARS}
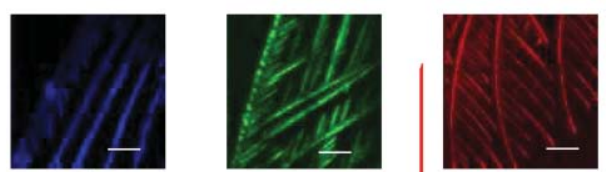

(b)

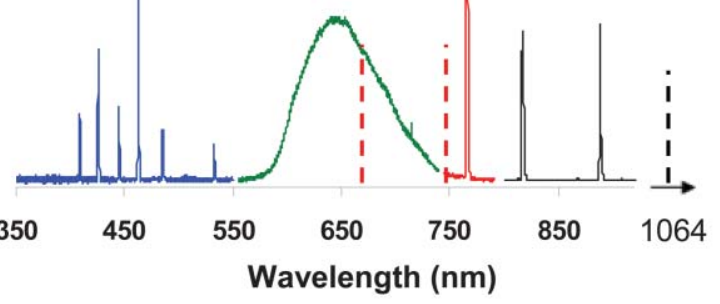

(c)

$662.8 \mathrm{~nm}$

$755.7 \mathrm{~nm}$
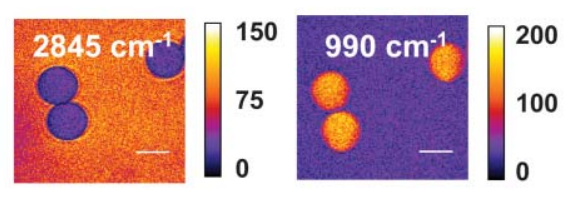

F-CARS ( $10^{3}$ counts $\left./ \mathrm{s}\right)$

Fig. 6 (a) Wave-mixing processes observed in nonlinear TTB crystals. Red: CARS processes; green: TPEF; dark blue: SFG; underlined blue: SHG. TTB sample as imaged with SFG, SHG, TPEF, and CARS. (b) OPOI: $816.8 \mathrm{~nm}$ (plain dark); OPO2: $888.7 \mathrm{~nm}$ (plain dark), and fundamental $1064 \mathrm{~nm}$ (dashed black) interact to generate CARS lines (red plain and dashed), a TPEF broad spectrum (green), and SHG and SFG lines (blue). (c) $250 \times 250$ pixel CARS images of $20 \mu \mathrm{m}$ polystyrene beads in olive oil for three Raman shifts $\left(2845 \mathrm{~cm}^{-1}\right.$, oil; $990 \mathrm{~cm}^{-1}$, polystyrene; $1855 \mathrm{~cm}^{-1}, \mathrm{NRB}$ ). Scale bar is $20 \mu \mathrm{m}$; pixel dwell time is $50 \mu \mathrm{s}$. Stokes and pump powers were 5 and $10 \mathrm{~mW}$, respectively. (Color online only.)

test sample for all these contrast mechanisms [Fig. 6(a)]. The spectrum depicted in Fig. 6(b) results from nonlinear interactions between the three wavelengths (OPOI: $816.8 \mathrm{~nm}$; OPO2: $888.7 \mathrm{~nm}$, and fundamenta11064 nm) in a TTB sample. Incoming beams (black) interact to generate CARS lines (red), a TPEF broad spectrum (green), and SHG and SFG lines (blue).

Focusing on the CARS contrast mechanism, three different Raman shifts can be advantageously used to image various chemical bonds. We illustrate this ability on Fig. 6(c) for $20 \mu \mathrm{m}$ polystyrene beads dispersed in olive oil. $2845 \mathrm{~cm}^{-1}$, $990 \mathrm{~cm}^{-1}, 1855 \mathrm{~cm}^{-1}$ Raman shifts are used to image oil, beads and the nonresonant background (NRB) respectively. Although the different contrast mechanisms have been illustrated on test TTB samples, they can be readily used for background free CARS and SHG imaging on fresh tissue samples.

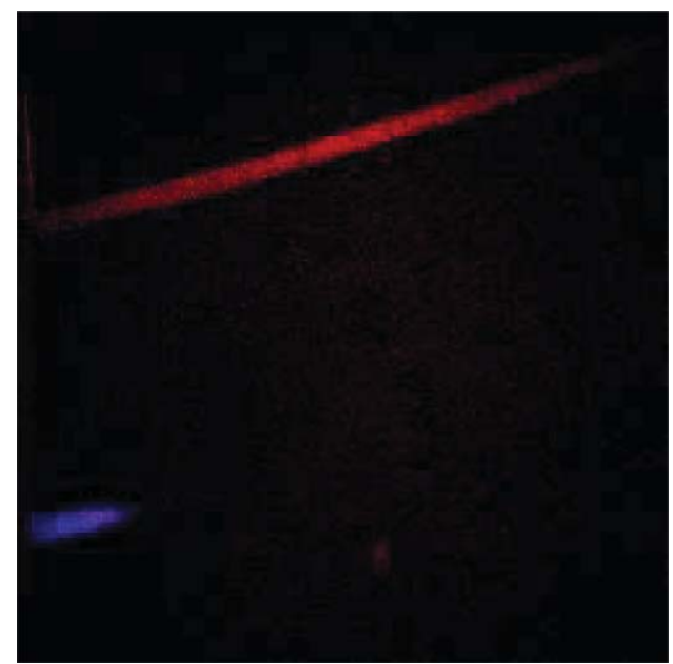

Video 1 Movie of the resonant CARS and SHG signals from an ear mouse on the $0-50 \mu \mathrm{m}$ depth range (QuickTime, 2.19 MB). [URL: http://dx.doi.org/10.1117/1.3533311.1]

\subsection{Tissue Imaging with Two Optical Parametric Oscillators}

One of the key features of this three-beam configuration is the ability to detect a "background-free" CARS signal as previously demonstrated. ${ }^{21,22}$ This is simply done by subtracting two CARS images that have been separately obtained. It should be noted here that this straightforward background subtraction is not free of artifacts and give satisfactory results only if the peak CARS signal is subtracted with a nonresonant background located far enough from the considered vibrational resonance. Although this is the case here, special care must be taken as reported in Ref. 23. Figure 7 illustrates such a scheme where the forward CARS anti-Stokes beam at $2845 \mathrm{~cm}^{-1}$ [Fig. 7(a), F-CARS R: lipid $\mathrm{CH}_{2}$ resonance] and $1855 \mathrm{~cm}^{-1}$ [Fig. 7(b), F-CARS NR: nonresonant] are acquired and subtracted (F CARS R-NR). In Fig. 7(e), this resonant CARS image (F-CARS R-NR) has been superimposed with the SHG image [E-SHG on Fig. 7(c) and associated spectrum Fig. 7(d)]. We attribute the CARS signal from lipids and the SHG's from collagen. One can clearly distinguish the lipid-rich sebaceous gland (4), one hair hole (2), and the stratum corneum (1); these lipid features are well separated from the collagen matrix (3).

Video 1 shows the resonant CARS and SHG images versus the penetration depths into the ear mouse sample. Quite noticeable is how the collagen matrix delineates the sebaceous gland near $z=30 \mu \mathrm{m}$.

Up to now, CARS images have been obtained for at most three wavenumbers (see Fig. 6). This might not be informative enough to tackle a specific molecular recognition problem. In Sec. 5, we investigate the possibility of performing hyper-spectral CARS imaging using OPOs.

\section{Hyper-Spectral Coherent Raman Spectroscopy with Two Optical Parametric Oscillators}

There has been only a few works on hyperspectral imaging in CRS, although it is well admitted that CRS imaging potentially has this ability. The first approach is known as " multiplex 


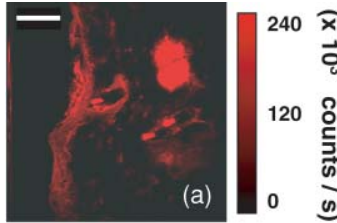

F-CARS R

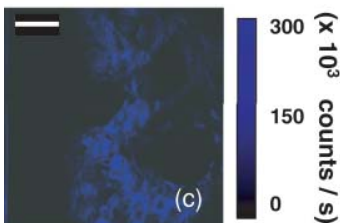

E-SHG

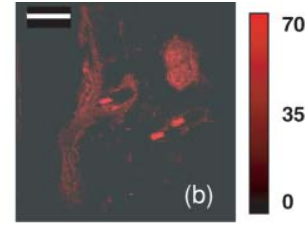

F-CARS NR $\times 10^{3}$

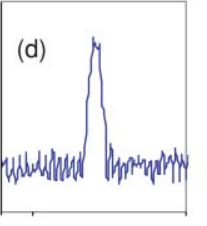

$400 \mathrm{~nm} \quad 420$

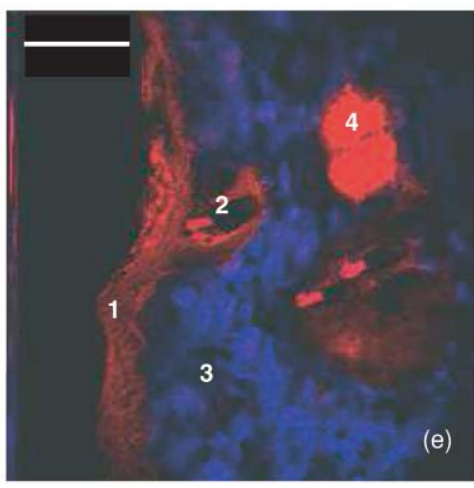

(F-CARS R-NR) + SHG

Fig. 7 Resonant CARS and SHG signals from a transversal skin mouse cryosection (20 $\mu \mathrm{m}$ thick). (a) Forward CARS $\mathrm{CH}_{2} 2845 \mathrm{~cm}^{-1}$, (b) Non resonant CARS $1855 \mathrm{~cm}^{-1}$, (c) SHG, and (d) associated spectrum. (e) (CARS $2845 \mathrm{~cm}^{-1}$ )-(CARS $1855 \mathrm{~cm}^{-1}$ ) difference super imposed with the SHG signal. 1: Stratum corneum; 2: hair hole; 3: collagen matrix; and 4: sebaceous gland. Scale bar is $40 \mu \mathrm{m}$; pixel dwell time is $50 \mu \mathrm{s}$; field of view is $200 \times 200 \mu \mathrm{m}^{2}(250 \times 250$ pixel); average is mo frames; Stokes and pump powers were 15 and $25 \mathrm{~mW}$, respectively.

CARS" and uses a broadband Stokes pulse and a narrowband pump pulse. ${ }^{24,25}$ The concept has evolved to more complex schemes involving a single broadband pulse that is temporally shaped. ${ }^{26-28}$ Although the multiplex approach has given impressive results in spectroscopy and microscopy, it ultimately faces the problem of sample photoinduced damage ${ }^{29}$ because the broadband spectrum carries rapidly too much power.

The second strategy, which is more straightforward, consists of scanning one of the two narrowband beams involved in the CRS process, the main problem being the rapid tunability of the narrowband source over a large spectrum. Although several attempts have been made that use a soliton self-frequency shift in photonic crystal fibers, ${ }^{30,31}$ these experiments remain at the level of a "proof of principle." More recently, a first demonstration of broadband CARS using a custom-made OPO has been reported in Ref. 32. Along the same line, we shall discuss here the possible use of commercial OPOs that can be fully automated to perform broadband hyperspectral imaging.

To achieve such a task, we shall use the two signal beams that are generated within two OPOs. Figure 8(a) presents the scheme of the experiment that is very similar to Fig. 5, except where the fundamental 1064-nm beam has been blocked. Figure 8(b) gives the pump (OPOI fixed at $735 \mathrm{~nm}$ ) and Stokes wavelengths (tuneable OPO2) associated with Raman shifts together with the corresponding anti-Stokes CARS wavelength. The present pump and Stokes wavelength configuration is similar to the one that can be found using two synchronized Ti:saphire lasers. ${ }^{1}$ It has the advantage of setting the CARS anti-Stokes wavelength at $<700 \mathrm{~nm}$, a spectral range wherein conventional photomultiplier tubes are very sensitive. For the detection, we use low-pass filters that enables one to record the anti-Stokes line while scanning the tuneable OPO2 over the $900-3200 \mathrm{~cm}^{-1}$ range.

To perform hyperspectral imaging, we use custom-made software that fully controls the OPO2 wavelength (see Sec. 2). This routine is included in the main nonlinear scanning microscope program to perform hyperspectral imaging. Figure 9 presents the result of a scan $\left(900-3150 \mathrm{~cm}^{-1}\right)$ range for a test $20-\mu \mathrm{m}$ polystyrene bead sample immersed in a nonresonant liquid (see Sec. 2). Figure 9(a) shows the sample imaged at $917 \mathrm{~cm}^{-1}$, corresponding to a nonresonant excitation.
Figure 9(b) presents a section of a single bead [along the dashed line of Fig. 9(a)] as a function of the Raman shift. This image is the ratio of the CARS signal from the bead to the CARS signal from the nonresonant liquid for each Raman shift. This simple operation is necessary because the OPO output power is not constant over the whole scanning range. Figure 9(c) depicts the raw

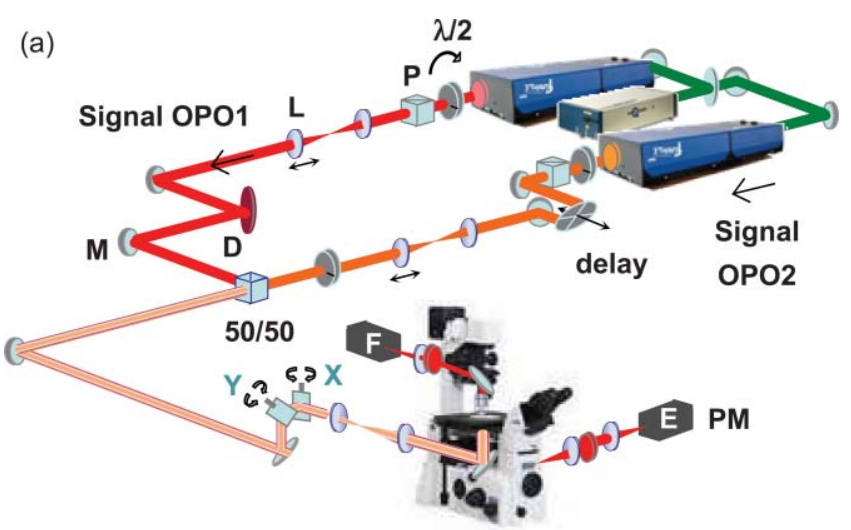

(b)

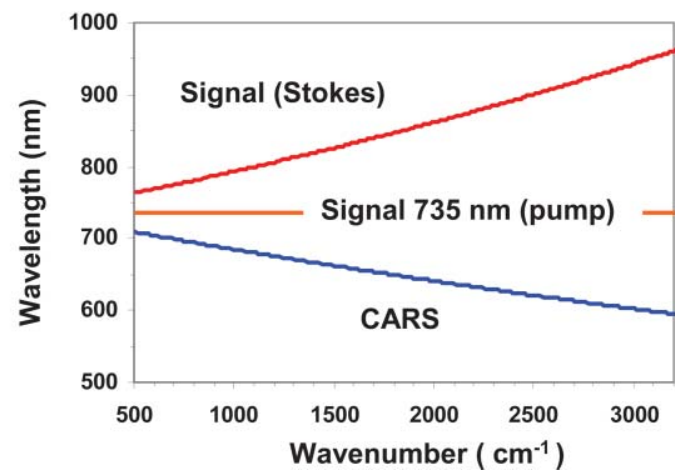

Fig. 8 Using two OPOs for hyperspectral imaging. (a) Scheme of the experiment where the two OPO signals are used. F and E stand for Forward and Epi (backward) detection; PM for photomultiplier tubes; $\mathrm{M}$ for silver mirrors; $\mathrm{P}$ for polarizer; $\mathrm{L}$ for NIR achromatic doublets, and D for dichroic filter. (b) OPOI set at $735 \mathrm{~nm}$ (pump), OPO2 signal (Stokes) and anti-Stokes CARS wavelengths associated to Raman shifts. 

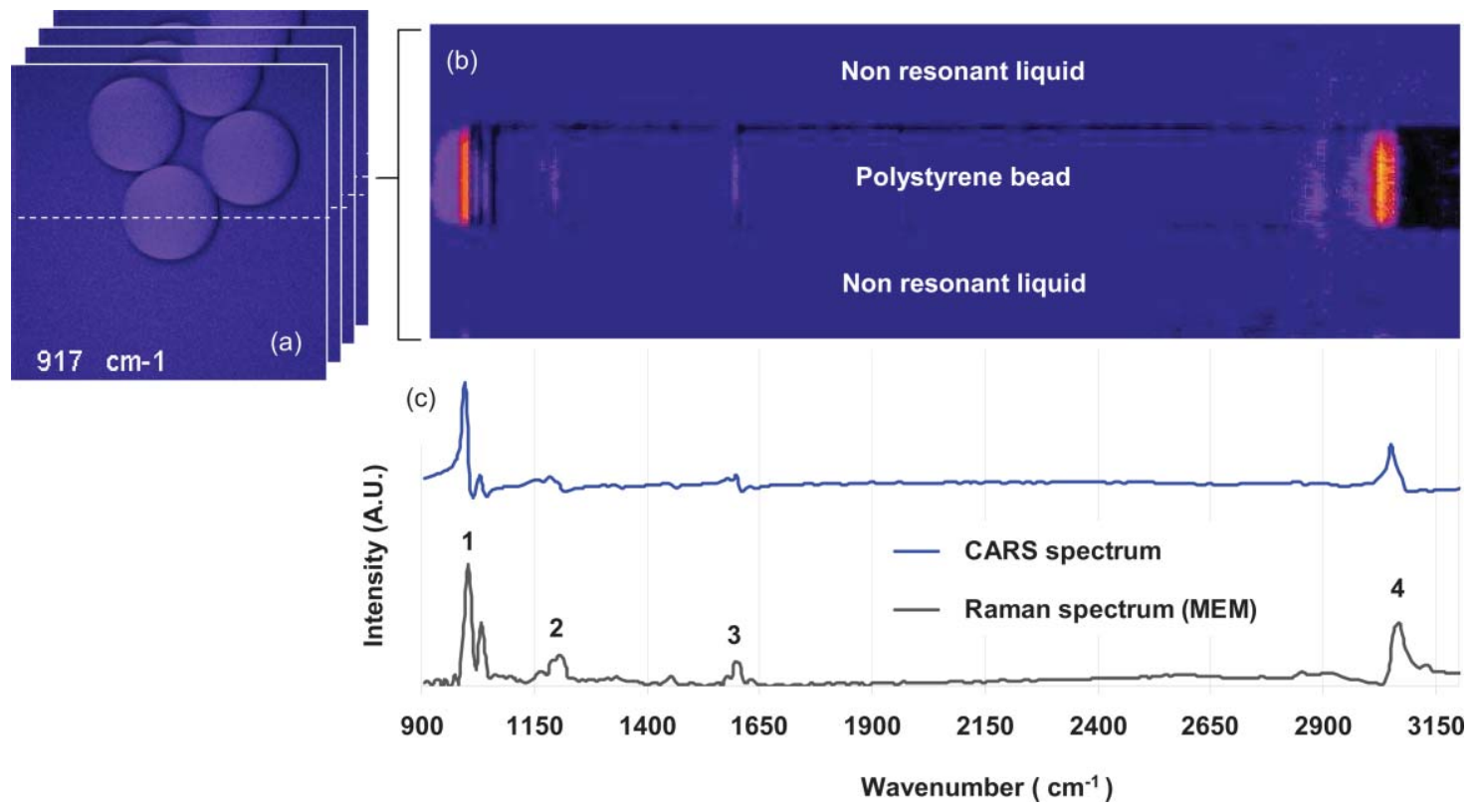

Fig. 9 Hyperspectral imaging of 20- $\mu$ m polystyrene beads. (a) Image at $917 \mathrm{~cm}^{-1}$ (NRB); (b) hyperspectral section of a single bead versus Raman shift; and (c) recorded CARS spectrum of the bead (upper curve) and processed with the maximum entropy method (MEM) (lower curve). Recovered Raman peaks 1: 1003 and $1033 \mathrm{~cm}^{-1} ; 2: 1158$ and $1188 \mathrm{~cm}^{-1} ; 3: 1584$ and $1604 \mathrm{~cm}^{-1}$; and 4: $3065 \mathrm{~cm}^{-1}$ Stokes and pump powers were $15 \mathrm{~mW}$.

CARS spectrum recorded on a single bead (upper curve) and the result of its processing with the maximum entropy method ${ }^{33}$ known to recover the pure Raman spectrum (lower curve). Most of the polystyrene spectral lines can be recovered from this spectrum, showing the effectiveness of the OPO scan. This scan uses 230 spectral points and was acquired for $60 \mathrm{~min}$ ( $15 \mathrm{~s}$ per spectral point). The main limitation comes from the temperature adjustment of the LBO crystal in the OPO that takes $\sim 10 \mathrm{~s}$.

Video 2 shows the hyperspectral image of the bead sample on the $900-3200 \mathrm{~cm}^{-1}$ range $\left(>2400 \mathrm{~cm}^{-1}\right.$, the contrast variation is due to strong variations in the reflection coefficient of the mirrors).

Hyperspectral imaging is implemented here in a quite sophisticated way using two OPOs but the OPO signal and fundamental $1064 \mathrm{~nm}$ configuration described in Sec. 3.2 would

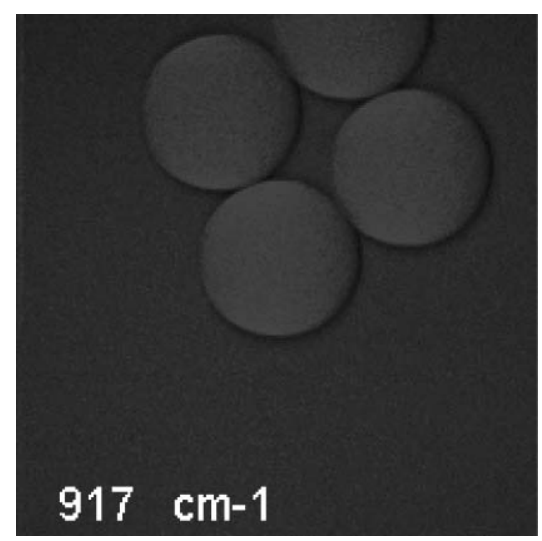

Video 2 Movie of the polystyrene beads sample on the $900-3200 \mathrm{~cm}^{-1}$ range (QuickTime, $4.75 \mathrm{MB}$ ).

[URL: http://dx.doi.org/10.1117/1.3533311.2] nicely do the job. In this case, the main limitation comes from the detection range of the photomultiplier tube that would practically limit the recorded spectra for Raman shifts of $>1500 \mathrm{~cm}^{-1}$.

\section{Conclusion}

This paper gives a snapshot of the possibilities offered by optical parametric oscillators to perform nonlinear microscopy with special emphasis on coherent Raman spectroscopy and microscopy. The one-OPO configuration is certainly the most cost-effective and efficient configuration to perform CRS. Together with the up-to-date high-grade microscope objective lens well corrected in the IR, using one OPO in the collinear configuration to perform CARS is just as simple as doing two-photon confocal microscopy. This is in strong contrast to the complicated CARS setup from a few years ago. Note that very recently, a compact system that includes, in the same box, the Nd:YVO pump and the OPO has been made available by APE. ${ }^{5}$ The system is taking care of the tuning, spatial, and temporal overlap, the power settings of both, the signal and fundamental $1064 \mathrm{~nm}$. It can also be used with signal and idler.

The two-OPO configuration remains expensive but offers flexibility and opens the route for higher order wave mixing. The current development in OPO is a great prospective for CRS (Refs. 34 and 35) and laser pumping OPO. ${ }^{36}$ The expected "shoe-box" CRS laser system will probably be available in the near future.

\section{Acknowledgments}

We thank Dr. Bong Rae Cho for synthesizing and providing us the TTB thin films, Dr. Ingo Rimke and Dr. Edlef Büttner (APE, Berlin) for valuable discussions, Dr. David Gachet for 
comments on the manuscript. We acknowledge financial support from the French Ministry of Research, the Centre National pour la Recherche Scientifique (CNRS), and the European Union (Grant CARSExplorer FP7 Health).

\section{References}

1. E. O. Potma, D. J. Jones, J.-X. Cheng, X. Xie, and J. Ye, "Highsensitivity coherent anti-Stokes Raman scattering microscopy with two tightly synchronized picosecond lasers," Opt. Lett. 27, 1168-1170 (2002).

2. C. L. Evans, E. O. Potma, M. Puoris'Haag, D. Côté, C. P. Lin, and X. S. Xie, "Chemical imaging of tissue in vivo with video-rate coherent anti-Stokes Raman scattering microscopy," Proc. Nat. Acad. Sci. USA 102, 16807-16012 (2005).

3. F. Ganikhanov, S. Carrasco, X. S. Xie, M. Katz, W. Seitz, and D. Kopf, "Broadly tunable dual-wavelength light source for coherent anti-Stokes Raman scattering microscopy," Opt. Lett. 31, 1292-1294 (2006).

4. HighQLaser, http://www.highqlaser.at/.

5. APE, http://www.ape-berlin. com/.

6. C. L. Evans, X. Xu, S. Kesari, X. S. Xie, S. T. Wong, and G. S. Young, "Chemically selective imaging of brain structures with CARS microscopy," Opt. Express 15, 12076-12087 (2007).

7. T. Hellerer, C. Axang, C. Brackmann, P. Hillertz, M. Pilon, and A. Enejder, "Monitoring of lipid storage in caenorhabditis elegans using coherent anti-Stokes Raman scattering (CARS) microscopy," Proc. Nat. Acad. Sci. USA 104, 14658-14663 (2007).

8. J. Moger, B. D. Johnston, and C. R. Tyler, "Imaging metal oxide nanoparticles in biological structures with CARS microscopy," Opt. Express 16, 3408-3419 (2008).

9. M. Zimmerley, C. Y. Lin, D. C. Oertel, J. M. Marsh, J. L. Ward, and E. O. Potma, "Quantitative detection of chemical compounds in human hair with coherent anti-Stokes Raman scattering microscopy," $J$. Biomed. Opt. 14, 044019 (2009).

10. E. Belanger, S. Begin, S. Laffray, Y. D. Koninck, R. Vallee, and D. Côté, "Quantitative myelin imaging with coherent anti-Stokes Raman scattering microscopy: alleviating the excitation polarization dependence with circularly polarized laser beams," Opt. Express 17, 18419-18432 (2009).

11. R. S. Lim, A. Kratzer, N. P. Barry, S. Miyazaki-Anzai, M. Miyazaki, W. W. Mantulin, M. Levi, E. O. Potma, and B. J. Tromberg, "Multimodal CARS microscopy determination of the impact of diet on macrophage infiltration and lipid accumulation on plaque formation in apoe-deficient mice," J. Lipid Res. 51, 1729-1737 (2010).

12. M. Windbergs, M. Jurna, H. L. Offerhaus, J. L. Herek, P. Kleinebudde, and C. J. Strachan, "Chemical imaging of oral solid dosage forms and changes upon dissolution using coherent anti-Stokes Raman scattering microscopy," Anal. Chem. 81, 2085-2091 (2009).

13. C. W. Freudiger, W. Min, B. G. Saar, S. Lu, G. R. Holtom, C. He, J. C. Tsai, J. X. Kang, and X. S. Xie, "Label-free biomedical imaging with high sensitivity by stimulated Raman scattering microscopy," Science 322, 1857-1861 (2008)

14. I. Rocha-Mendoza, W. Langbein, P. Watson, and P. Borri, "Differential coherent anti-Stokes Raman scattering microscopy with linearly chirped femtosecond laser pulses," Opt. Lett. 34, 2258-2260 (2009).

15. H. Chen, H. Wang, M. N. Slipchenko, Y. Jung, Y. Shi, J. Zhu, K. K. Buhman, and J. X. Cheng, "A multimodal platform for nonlinear optical microscopy and microspectroscopy," Opt. Express 17, 1282-1290 (2009).

16. J.-X. Cheng, A. Volkmer, L. D. Book, and X. Xie, "An epi-detected coherent anti-Stokes Raman scattering (E-CARS) microscope with high spectral resolution and high sensitivity," J. Phys. Chem. B 105, 12771280 (2001).
17. M. Y. Jeong, H. M. Kim, S. J. Jeon, S. Brasselet, and B. R. Cho, "Octupolar films with significant second-harmonic generation," Adv Mater. 19, 2107-2111 (2007).

18. P. Ferrand, M. Pianta, A. Kress, A. Aillaud, H. Rigneault, and D. Marguet, "A versatile dual spot laser scanning confocal microscopy system for advanced fluorescence correlation spectroscopy analysis in living cell," Rev. Sci. Instrum. 80, 83702 (2009).

19. Hamamatsu (Japan) PM R3896 (1\% quantum effciency at $880 \mathrm{~nm}$ ); Perkin Elmer (USA) C1372 (1\% quantum effciency at $830 \mathrm{~nm})$.

20. A. Volkmer, L. D. Book, and X. S. Xie, "Time-resolved coherent antiStokes Raman scattering microscopy: imaging based on Raman free induction decay," App. Phys. Lett. 80, 1505-1507 (2002).

21. F. Ganikhanov, C. L. Evans, B. G. Saar, and X. S. Xie, "High-sensitivity vibrational imaging with frequency modulation coherent anti-Stokes Raman scattering (FM CARS) microscopy," Opt. Lett. 31, 1872-1874 (2006).

22. O. Burkacky, A. Zumbusch, C. Brackmann, and A. Enejder, "Dualpump coherent anti-Stokes-Raman scattering microscopy," Opt. Lett. 31, 3656-3658 (2006).

23. C. Fang, F. Lu, W. Zheng, and Z. Huang, "Triple-frequency symmetric subtraction scheme for nonresonant background suppression in coherent anti-stokes raman scattering (cars) microscopy," Opt. Express 18, 15714-15724 (2010)

24. M. Müller and J. M. Schins, "Imaging the thermodynamic state of lipid membranes with multiplex CARS microscopy," J. Phys. Chem. B $\mathbf{1 0 6}$ 3715-3723 (2002).

25. J. X. Cheng, A. Volkmer, L. D. Book, and S. Xie, "Multiplex coherent anti-Stokes Raman scattering microspectroscopy and study of lipid vesicles," J. Phys. Chem. B 106, 8493-8498 (2002).

26. N. Dudovich, D. Oron, and Y. Silberberg, "Single-pulse coherently controlled nonlinear Raman spectroscopy and microscopy," Nature $\mathbf{4 1 8}$ 512-514 (2002).

27. D. Oron, N. Dudovich, and Y. Silberberg, "Femtosecond phase-andpolarization control for background-free coherent anti-Stokes Raman spectroscopy," Phys. Rev. Lett. 90, 213902 (2003).

28. B. C. Chen and S. H. Lim, "Optimal laser pulse shaping for interferometric multiplex coherent anti-Stokes Raman scattering microscopy," J. Phys. Chem. B 112, 3653-3661 (2008).

29. Y. Fu, H. Wang, R. Shi, and J.-X. Cheng, "Characterization of photodamage in coherent anti-Stokes Raman scattering microscopy," Opt. Express 14, 3942-3951 (2006).

30. E. R. Andresen, H. N. Paulsen, V. Birkedal, J. Thgersen, and S. R. Keiding, "Broadband multiplex coherent anti-stokes raman scattering microscopy employing photonic-crystal fibers," J. Opt. Soc. Am. B 22, 1934-1938 (2005).

31. K. Tada and N. Karasawa, "Broadband coherent anti-Stokes Raman scattering spectroscopy using soliton pulse trains from a photonic crystal fiber," Opt. Commun. 282, 3948-3952 (2009).

32. P. F. Chimento, M. Jurna, H. S. P. Bouwmans, E. T. Garbacik, L. Hartsuiker, C. Otto, J. L. Herek, and H. L. Oflerhaus, "High-resolution narrowband CARS spectroscopy in the spectral fingerprint region," $J$. Raman Spectrosc. 40, 1229-1233 (2009).

33. E. M. Vartiainen, H. A. Rinia, M. Müller, and M. Bonn, "Direct extraction of Raman line-shapes from congested CARS spectra," Opt. Express 14, 3622-3630 (2006).

34. B. G. Saar, G. R. Holtom, C. W. Freudiger, C. Ackermann, W. Hill, and X. S. Xie, "Intracavity wavelength modulation of an optical parametric oscillator for coherent Raman microscopy," Opt. Express 17, 1253212539 (2009)

35. M. Jurna, J. P. Korterik, C. Otto, J. L. Herek, and H. L. Ofierhaus, "Vibrational phase contrast microscopy by use of coherent anti-Stokes Raman scattering," Phys. Rev. Lett. 103, 043905 (2009).

36. K. Kieu, B. G. Saar, G. R. Holtom, X. S. Xie, and F. W. Wise, "Highpower picosecond fiber source for coherent Raman microscopy," Opt. Lett. 34, 2051-2053 (2009). 